\title{
Zero-Free-Parameter Modeling Approach to Predict the Voltage of Batteries of Different Chemistries and Supercapacitors under Arbitrary Load
}

\author{
Elif Özdemir, Can Berk Uzundal, and Burak Ulgut ${ }^{*, z}$ \\ Department of Chemistry, Bilkent University, 06800 Ankara, Turkey
}

Performance modeling of electrochemical energy storage systems is gathering increasingly higher attention in recent years. With the ever increasing power demand of mobile applications, predicting voltage behavior under different load profiles is of utmost importance for communications, automotive and consumer electronics. The ideal modelling approach needs not only to accurately predict the response of the battery, but also be robust, easy to implement and have low computational complexity. We will present a new algorithm that is algebraically straightforward, that has no adjustable parameters and that can accurately predict the voltage response of batteries and supercapacitors. The approach works well in a variety of discharge profiles ranging from simple long DC discharge/charge profiles to pulse schemes based on drive schedules published by regulatory bodies. Our approach is based on Electrochemical Impedance Spectroscopy measurements done on the system to be predicted. The spectrum is used in the frequency domain without any further processing to predict the fast moving portion of the voltage in the frequency domain. DC response is added in through a straightforward lookup table. This widely applicable approach can predict the voltage of with less than $1 \%$ error, without any adjustable parameters to a large variety of discharge profiles.

(C) 2017 The Electrochemical Society. [DOI: 10.1149/2.1521706jes] All rights reserved.

Manuscript submitted February 15, 2017; revised manuscript received March 30, 2017. Published April 15, 2017.

Battery $^{\mathrm{a}}$ performance in a variety of conditions is one of the most crucial design criteria in modern consumer electronics, electric vehicles and various defense related equipment. The parameters of the battery generally limit not only operation time between charge cycles, but also size of the finished product. Charge capacity of the battery is the first important specification that controls the lifetime and size of the finished product. However, a simple capacity estimation does not provide the complete picture. Battery capacity is specified under very specific conditions that are mostly at constant current. The exact value of this current, how the battery is charged beforehand and how long the charge and the discharge will proceed depend heavily on the chemistry and the standards (and conventions) that are developed for the system in question. However, in real life applications, the discharge demands on the battery is far from a constant current discharge. ${ }^{1,2}$ Behavior of the battery under the non-uniform discharge conditions needs to be determined, understood and accounted for, in order to achieve the best design and choice for any application. With the ever-increasing number of mobile powered applications, an accurate and easy to implement modeling approach to evaluate various batteries is of utmost necessity. The vast number of different discharge/charge use-cases makes measurement of all possible discharge profiles distinctly impractical.

In a recent review Fotouhi et al. $^{3}$ covered various perspectives of understanding and predicting battery behavior based on different assumptions. As we will discuss below, all methods have their advantages and drawbacks. Methods with low computational complexity tend to have high inaccuracies whereas methods that are accurate tend to be algebraically and computationally complex.

In another recent review, Abada et al. ${ }^{4}$ covered battery modeling approaches as they relate to the safety considerations of batteries. Understanding and predicting battery behavior is crucial for not only the performance of the system, but also, the safety of the products. Most battery accidents encountered recently are either due to mishandling of the battery that leads to some physical deformation ${ }^{5}$ or they are caused by the battery getting charged or discharged to potentials that are not safe for its chemistry. ${ }^{6}$ In accidents of the latter kind, the battery voltage predictions to be accurate are of great importance.

Battery voltage is the main response of the battery as a function of applied current profiles. The fundamental parameter dictating the battery voltage response is the state-of-charge (SOC). In any battery chemistry, an electrochemical potential exists as a function of the activities of the various electroactive species. This potential, in theory, can be defined using the appropriate combination of the relevant group of Nernst Equations. ${ }^{7}$ Electrochemical potential provides the basis for any modeling approach. ${ }^{8}$ However, it only reflects the thermodynamic equilibrium condition and does not capture the response of the batteries to current spikes or oscillating currents. Therefore, the $\mathrm{AC}^{\mathrm{b}}$ response of the battery needs to be modeled in addition to the $\mathrm{DC}^{\mathrm{c}}$ response. The sum of the $\mathrm{AC}$ response and the $\mathrm{DC}$ response yields the actual real-time battery voltage.

In recent years, a number of different approaches to battery performance modeling have been developed that takes into account the deviations from the electrochemical equilibrium potential. These have been reviewed by Nejad et al. ${ }^{9}$ All methods that are employed require fundamental parameters that explain the various electrochemical interfaces and the chemical and electrochemical reactions within the battery. Kinetics and thermodynamics of the reactions of the battery, along with the double layer charging of the anode/electrolyte interface, cathode/electrolyte interface and potentially the dielectric of the separator need to be explained in a proper model for the battery.

In addition to the kinetics and thermodynamics of the reactions and the various capacitive charging phenomena, transport is another limiting issue that needs to be incorporated into an accurate model. Transport to and from well-defined geometries are well characterized and are solvable analytically. However, batteries, like any other electrochemical energy storage system (EES), rely on the electrode/electrolyte interface to function. Since the charge storage is interface based, a high surface area is beneficial. This high surface area is accomplished through porous electrodes. Though porous electrodes are advantageous in order to increase the interfacial area, they severely complicate the ionic transport phenomena. Since the pores are so numerous, they are impossible to individually characterize and catalog in terms of shapes, sizes and their surface chemistry.

Further, the active materials that are commonly used are electronically insulating especially in Li based battery chemistries. ${ }^{10,11}$ Therefore, the electrode structure is made up of a mixture of the insulating, yet electrochemically active intercalation material, some carbonaceous conducting additive and a binder to hold everything together. This structure has a very complicated electronic transport 
behavior in addition to the very complicated ionic transport through the various pores.

For all the reasons mentioned, practically, these parameters are impossible to obtain individually in an accurate fashion. Most of the time, the kinetic and transport parameters that explain the behavior of the systems, or implications of groups of said parameters are measured through electrochemical impedance spectroscopy (EIS). EIS measurements are made on batteries and fit to equivalent circuit models that explain the various parameters to differing levels of detail. In the simplest case, the data is fit to a simplified Randles cell ${ }^{12}$ that lumps all the capacitive interfaces into a single capacitor and all the kinetic terms into a single resistor that is put in parallel to this capacitor while, all the transport phenomena are lumped into a single resistor. Though this impedance model and the voltage profile predicted through it captures the fundamental properties of the response, it lacks the fine detail in the data. In the opposite extreme, porous interfaces are modeled using detailed transmission lines and the rest of the interfaces are modeled with constant phase elements and capacitors along with the resistors that explain the kinetic and transport phenomena. ${ }^{13,14}$

Even though the accuracy of the individual kinetic and thermodynamic parameters are questionable, modeling approaches that start from first principles are reported. In one example Fan et al. ${ }^{15}$ developed a model that includes the thermodynamic half-cell potentials that are corrected by solid state diffusion, the Butler-Volmer kinetics for the electrode reactions, liquid phase diffusion for either side of the system along with the ohmic drop. Using this formulation, the authors developed a decent understanding of the internal operation of the system and followed the concentrations of various species within the system. In another study, Verbrugge et al. ${ }^{16}$ reported a formulation that explains the slow transport phenomena associated with a $\mathrm{Li}-\mathrm{Si}$ anode in detail. Using their model, they could follow and predict the concentration profiles and the speciation of $\mathrm{Li}$ species inside the $\mathrm{Si}$ anode.

One straightforward way to model the performance of batteries is to take the equivalent circuit model and use the same components in the time domain to predict the fast moving part of the response. This approach was shown to be useful by Barsoukov et al. ${ }^{14}$ where simple SPICE simulations were conducted using non-linear equivalent circuits that were developed for the cells in question. More recently, a similar approach was also shown to work by Castano et al. ${ }^{12}$ where a simplified Randles cell was used in order to predict the battery behavior. In a different study, ${ }^{17}$ Thele et al. presented a method based on impedance spectroscopy and included a term to explain the hysteretic behavior of NiMH type batteries. Mentioned references show that this type of an approach can reproduce the voltage profiles, as accurately as the equivalent circuit model parameters reproduce the impedance spectrum.

Simple models such as the simplified Randles model clearly fail to capture all the details of EIS data. However, taking the more complicated models into time domain complicate the algebra and increase greatly the relevant computation time for the modeling. ${ }^{18}$

We are presenting a new approach that combines the best of both worlds. Instead of modeling the impedance spectra using an equivalent circuit model that is either incomplete or very complicated, we will present an approach to use the impedance data as is. Our approach makes no assumptions and has no fit parameters, instead, it uses the measured impedance spectrum in order to predict the response directly.

Instead of trying to obtain parameters from impedance spectra in order to build a response function in the time domain, we are doing the modeling in the frequency domain for the AC part of the response. A Fourier transform of the desired current profile yields the frequency domain current profile, this frequency domain current profile is simply multiplied by the impedance at every frequency contained by the transform. This yields the $\mathrm{AC}$ voltage response of the battery as a function of frequencies that are relevant to the profile. Finally, a simple inverse Fourier transform of the voltage profile yields the voltage response of the batteries in the time domain. The DC

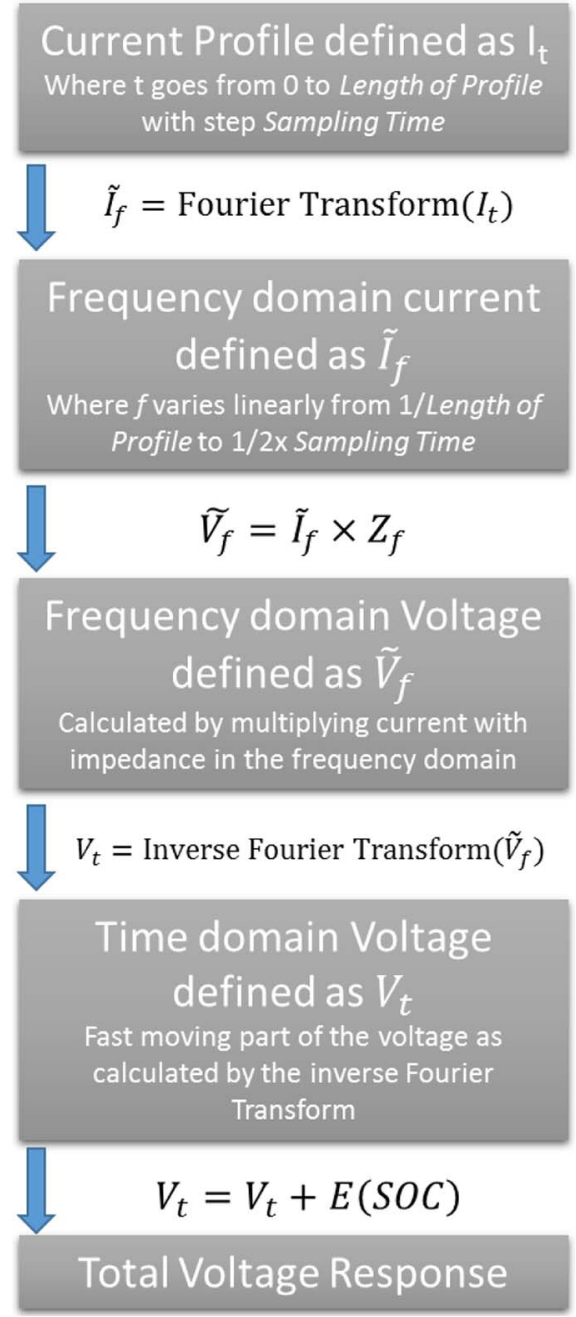

Figure 1. Steps of the methodology.

response of the battery is then added in through a simple map of SOC vs DC potential obtained experimentally.

As shown in Figure 1, this approach is computationally quite simple and very straightforward to implement. The steps are all doable analytically and do not involve any approximations or assumptions.

\section{Experimental}

In order to test the validity of our approach, two kinds of batteries with different chemistries $\left(\mathrm{LiFePO}_{4}\right.$ and $\left.\mathrm{NiMH}\right)$ and a supercapacitor was chosen to be representative of different EES. Going further, abbreviations are going to be used instead of the full name of the samples studied. IFR $22650 \mathrm{LiFePO}_{4}$ battery from EWT Battery from Shenzhen, China, will be denoted by LFP, $1200 \mathrm{mAh}$ NiMH battery from GPI International Limited from Hong Kong will be denoted by NiMH and BCAP0350, a $350 \mathrm{~F}$ ultracapacitor from Maxwell Technologies, USA, will be denoted by $350 \mathrm{~F}$. Samples of these devices were obtained from local wholesalers.

Gamry Interface 5000E Potentiostat/Galvanostat/ZRA was employed for measuring impedance spectra, DC potential vs. SOC data and the response to the discharge profile chosen. For arbitrary current profiles used in this study, along with impedance spectra at relevant frequency values, customs scripts were written in-house using EXPLAIN language used by the Gamry Framework software.

The DC voltage vs. SOC map were obtained using a DC current discharge, at a DC current that is adjusted such that the current is $0.1 \%$ of the numerical capacity of the battery (e.g $1.2 \mathrm{~mA}$ for the 
$1200 \mathrm{mAh}$ NiMH battery). For the 350F, this was measured by $3.5 \mathrm{~mA}$. This current is intentionally chosen to be small since a slow discharge is preferred for the potential to accurately reflect the equilibrium potential at the various SOC. A small current ensures that the contributions from non-equilibrium effects are small enough to be ignored.

EIS data were gathered in potentiostatic mode with $1 \mathrm{mV} \mathrm{AC}$ amplitude. Potentiostatic measurement ensures that the battery voltage does not drift throughout the measurement and $1 \mathrm{mV}$ AC amplitude ensures linearity of the measured data. The linearity and the stability are verified through a Kramers-Kronig (KK) test using the built-in KK transform test in Echem Analyst. The impedance data, along with the KK tests are shown in the supporting information figure S2. Since the data is gathered potentiostatically to ensure stability, and $1 \mathrm{mV}$ is chosen to ensure linearity, the KK transforms fit perfectly to the data without any noticeable deviation. The $\chi^{2}$ value for all fits are less than $2 \times 10^{-6}$.

Frequencies that are used are linearly spaced and not logarithmically spaced which is generally the case in EIS. The log spacing ensures proper coverage of all processes within a system, however, in the present case, frequencies required are dictated by the length and the sampling time of the given discharge profile. Therefore, for every profile measured, a distinct set of frequencies were measured using a modified version of the custom script.

In between every measurement, the battery was charged to the voltage chosen for the specific system (3.340 V and 3.120 V for LFP, $1.240 \mathrm{~V}$ for NiMH and $1.500 \mathrm{~V}$ for $350 \mathrm{~F}$ ). This voltage was chosen such that the system under test was not operating at charge/discharge extremes and had minimal side reactions during charge or discharge. The charging was performed using a constant potential hold for 10 hours in order to ensure equilibration of the system at the potential of interest. This ensured that any leftover relaxation from the constant current charging process decayed to below the noise level before the start of the test.

\section{Modeling Methodology}

The modeling methodology is broken into two separate but equally important parts given the discharge profiles. The AC part of the response is calculated in the frequency domain based on the impedance spectrum of the system to be modeled. The DC part is added in an additional step, using a simple map of voltage as a function of SOC, which is commonly done in the literature. ${ }^{9}$

The AC part of the voltage response is calculated based on a frequency domain complex multiplication. The arbitrary current profile is Fourier transformed to yield the current as a function of frequency along with the corresponding frequencies. In order to calculate the voltage at the frequencies of interest, impedance values at all required frequencies are measured. Complex impedance values are then multiplied by complex frequency domain current values to yield an expected complex voltage response in the frequency domain. At this point, a simple inverse Fourier transform is performed to obtain the fast moving part of the time domain voltage response.

The DC part of the voltage is then added using a map of SOC vs. DC voltage. This map is measured using a low current discharge as explained in the Experimental section. In order to employ this map, the overall charge $(\mathrm{Q})$ as a function of any time point $(\mathrm{j})$ in the discharge profile is calculated by a sum of current(I) of the profile multiplied by time intervals $(\Delta t)$ at all points(i) up to the time point (j).

$$
Q(j)=\sum_{i=1}^{j} I(i) \times \Delta t
$$

The resulting charge values are then matched to corresponding voltage values in the map of SOC vs. DC voltage. The DC voltages are added to the fast moving part calculated above to yield the final result.

Computations along with the complex multiplications were done using arrays defined through NumPy v1.9.2 and Fourier transforms were done within SciPy v0.5.11. Spyder v2.3.5.2 was used as the development environment.

\section{Test Cases}

As test cases for the methodology, two profiles published by Environmental Protection Agency (EPA) ${ }^{19}$ are used. Specifically, the profiles chosen are Highway Fuel Economy Test Drive Schedule

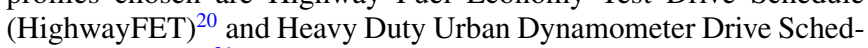
ule (HDUDDS). ${ }^{21}$ These profiles are just two of a long list that are published by EPA along with other regulatory bodies in order to standardize the testing procedures for automotive applications. The profiles chosen have both high acceleration and deceleration levels, in addition to long flat regions to demonstrate the versatility of our approach.

These profiles are published as arrays of speeds at various time points. In order to convert these speeds to current demand from the battery, the following procedure is applied. First, accelerations are calculated as simple two point finite differences. Then, accelerations are normalized to have the highest acceleration level to be unity. In a final step, decelerations that lead to charging of the batteries are multiplied by $40 \%$ in order to account for inefficiencies in regenerative braking ${ }^{\mathrm{d}}$. In the final step, power is assumed to be directly proportional to the current that is drawn from the energy storage system. This implies that the voltage of the battery does not change appreciably throughout the test. Though this assumption is somewhat valid for a battery based system, it is certainly not valid for a supercapacitor. However, since the particulars of the actual discharge profiles is immaterial for the present work, this point is not considered further ${ }^{\mathrm{e}}$.

In addition, one profile involving a relatively long charge and a relatively long discharge step followed by a rest was used. Since the method is based on impedance spectroscopy, this profile was chosen in order to test the validity of the approach in profiles that involve slowly changing currents. The length and the sampling rate of this profile is adjusted to coincide with those of HDUDDS in order to have the set of frequencies correspond to those of the said profile. This profile will be referred to as a Squarewave as an abbreviation.

The above mentioned profiles thus calculated are used as a templates for every battery. The amplitude of the profile is then adjusted according to the battery capacity. For batteries, this current amplitude was chosen to be $10 \%$ of the numerical capacity in Ah of the battery for both batteries and all three profiles, except Squarewave for LFP. This amplitude was chosen to be $1 \%$ of the numerical capacity to check linearity. For the supercapacitor, experiments were performed at currents that are chosen to be the numerical value of the capacitance in Farads in mA. Finally, in order to check for a deep discharge scenario, the profiles were scaled by $2.2 \mathrm{~A}$ for the LFP case in one of the tests. As examples, the above mentioned profiles, scaled to $350 \mathrm{~mA}$ are shown in Figure 2.

\section{Results and Discussion}

Three types of samples studied have distinctly different impedance spectra in the relevant frequency region. These spectra are shown in supplementary information figure $\mathrm{S} 2$. The $350 \mathrm{~F}$ shows a distinctly more capacitive spectrum whereas two battery systems chosen show a more resistive character. Differences in the EIS data are only relevant to the present study in showing the wide applicability of the model and they are presented as evidence of the said issue. Reasons behind the differences of the spectra are well understood and will not be discussed here.

Figures 3, 4, 5 show comparisons of the calculated voltage and the measured voltage overlaid on each other for LFP, 350F and NiMH respectively with all three discharge profiles studied. In all cases, the

\footnotetext{
${ }^{\mathrm{d}}$ This number fluctuates a lot in the literature and there is probably no hard single number on the efficiency. However, $40 \%$ was chosen as an average for the current study.

${ }^{\mathrm{e}}$ The data, along with a summary of the steps is shown in supporting information figure $\mathrm{S} 1$.
} 

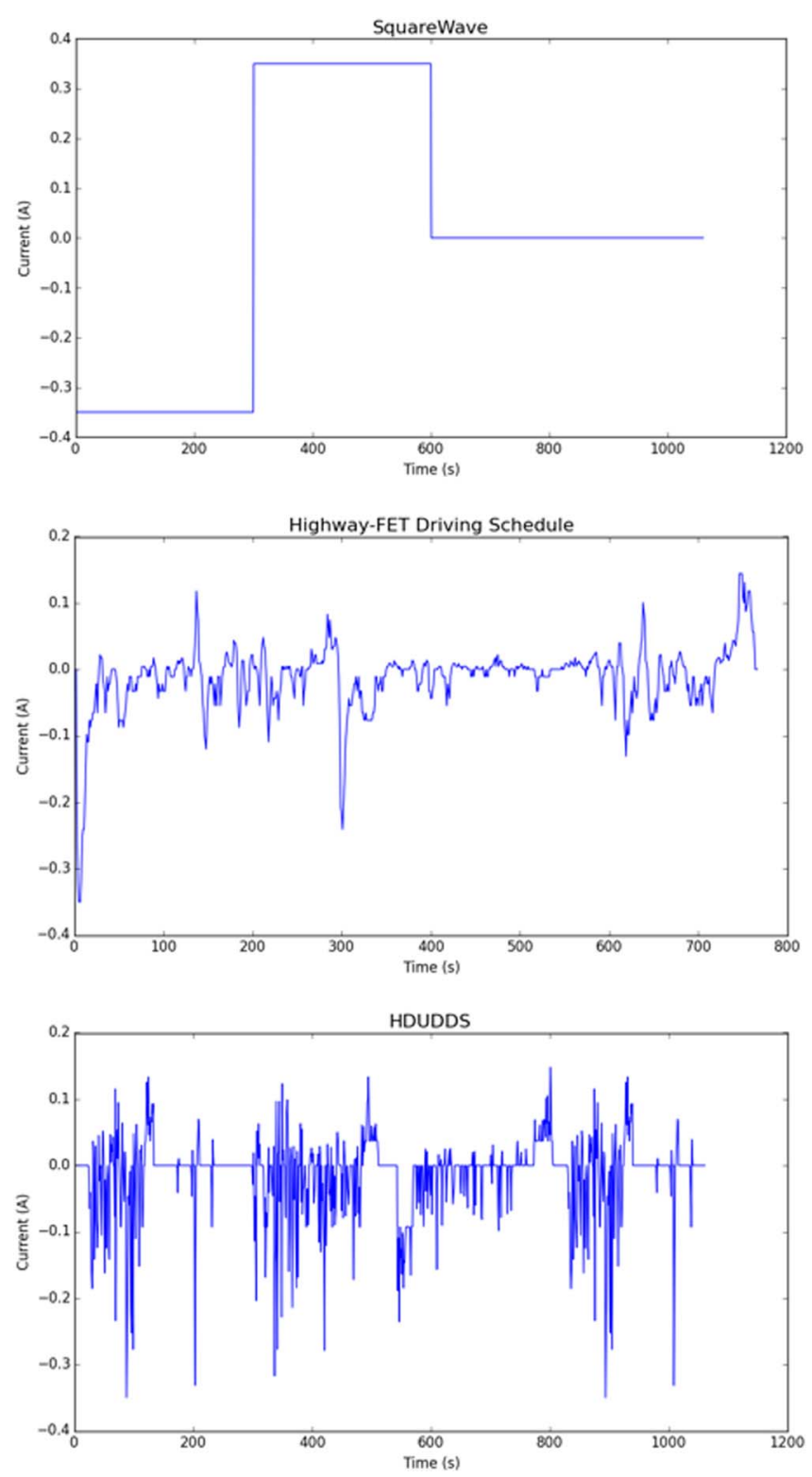

Figure 2. The current profiles used in this work as an example, $350 \mathrm{~mA}$ amplitude profiles are shown that were used for the $350 \mathrm{~F}$.

prediction error is less than $1 \%$. In the HDUDDS and Highway FET profiles, errors are much less than $1 \%$ as shown in Table I.

The success of the predictions is impressive given the algorithm has no adjustable parameters and is only based on measurements of DC Voltage vs. SOC and a proper impedance spectrum. Using these two datasets and nothing else, the response of the battery is predicted with a model that relies on straightforward algebra and with minimal computational complexity. This model can be used to predict the performance of batteries under arbitrary load without any adjustable parameters.

Table I. Error table of three different systems with three different profiles.

\begin{tabular}{llll} 
& LFP & NiMH & $350 \mathrm{~F}$ \\
\hline HighwayFET & $<0.08 \%$ & $<0.33 \%$ & $<0.04 \%$ \\
HDUDDS & $<0.08 \%$ & $<0.22 \%$ & $<0.03 \%$ \\
Squarewave & $<0.03 \%$ & $<0.91 \%$ & $<0.92 \%$
\end{tabular}
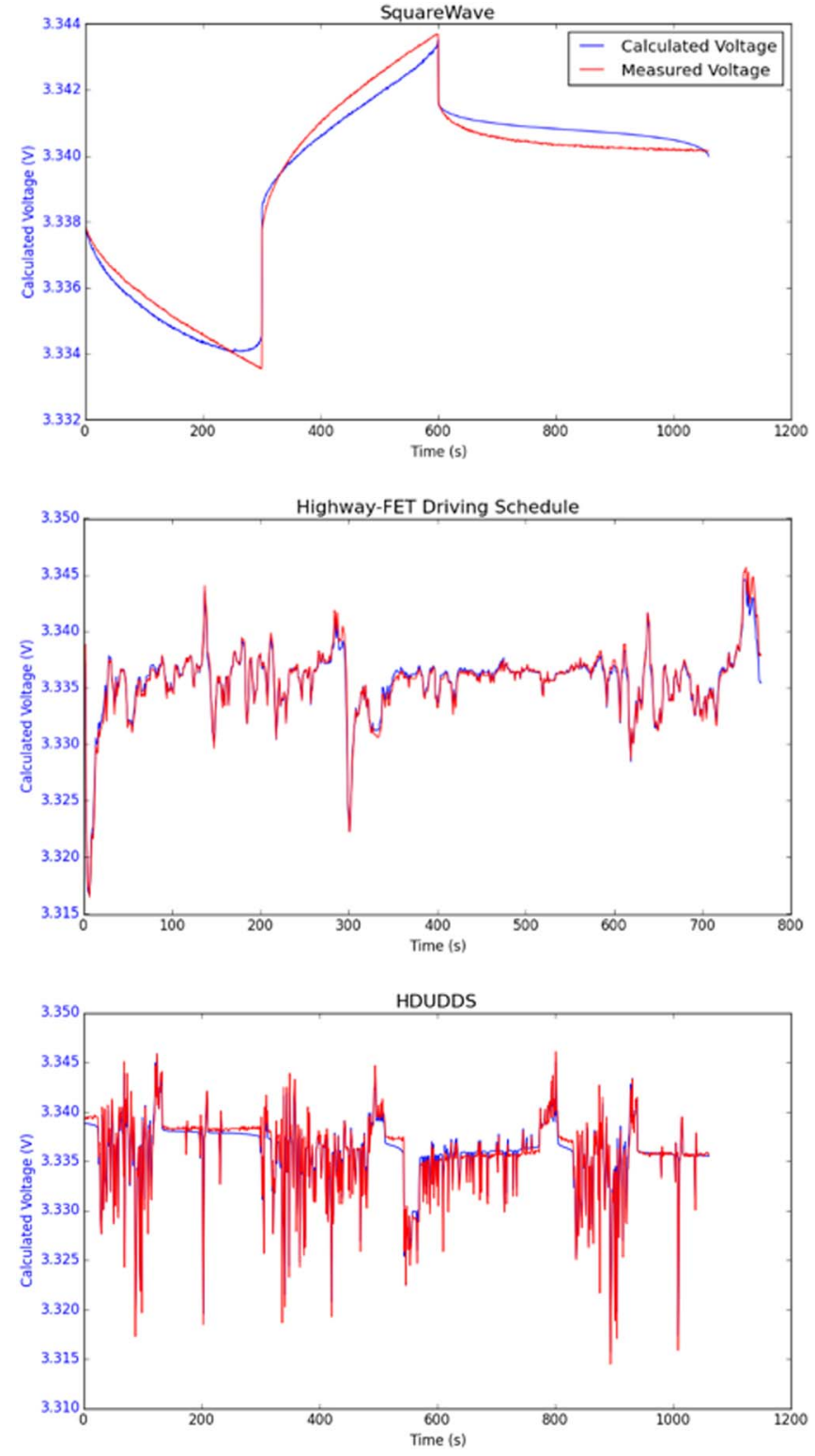

Figure 3. The response of LFP (in red) and the predicted value (in blue) for three profiles. Squarewave applied at $22 \mathrm{~mA}$ and both Highway-FET and HDUDDS applied at $220 \mathrm{~mA}$.

Below, we will discuss our various results and compare the effects of certain issues on the performance of the predictive power of the approach.

Details of the energy storage chemistry/mechanism.-The approach presented in the current work has no implications or assumptions regarding the underlying mechanism of the electrochemistry of energy storage. As the impedance spectrum is measured and is used directly without any fits or modeling, the details of neither the impedance spectrum, nor the electrochemistry within compromise the wide applicability of the model.

Since the approach presented only relies on the measured parameters through impedance spectroscopy and a slow discharge without any assumptions about neither, it is expected to work irrespective of the sample. The NiMH, LFP and the 350F are modeled equally as well with minimal error. This clearly shows that the approach is fundamentally sound and can predict the voltage response of the system. As demonstrated by the successful predictions across different systems with different electrochemical energy storage chemistries and 

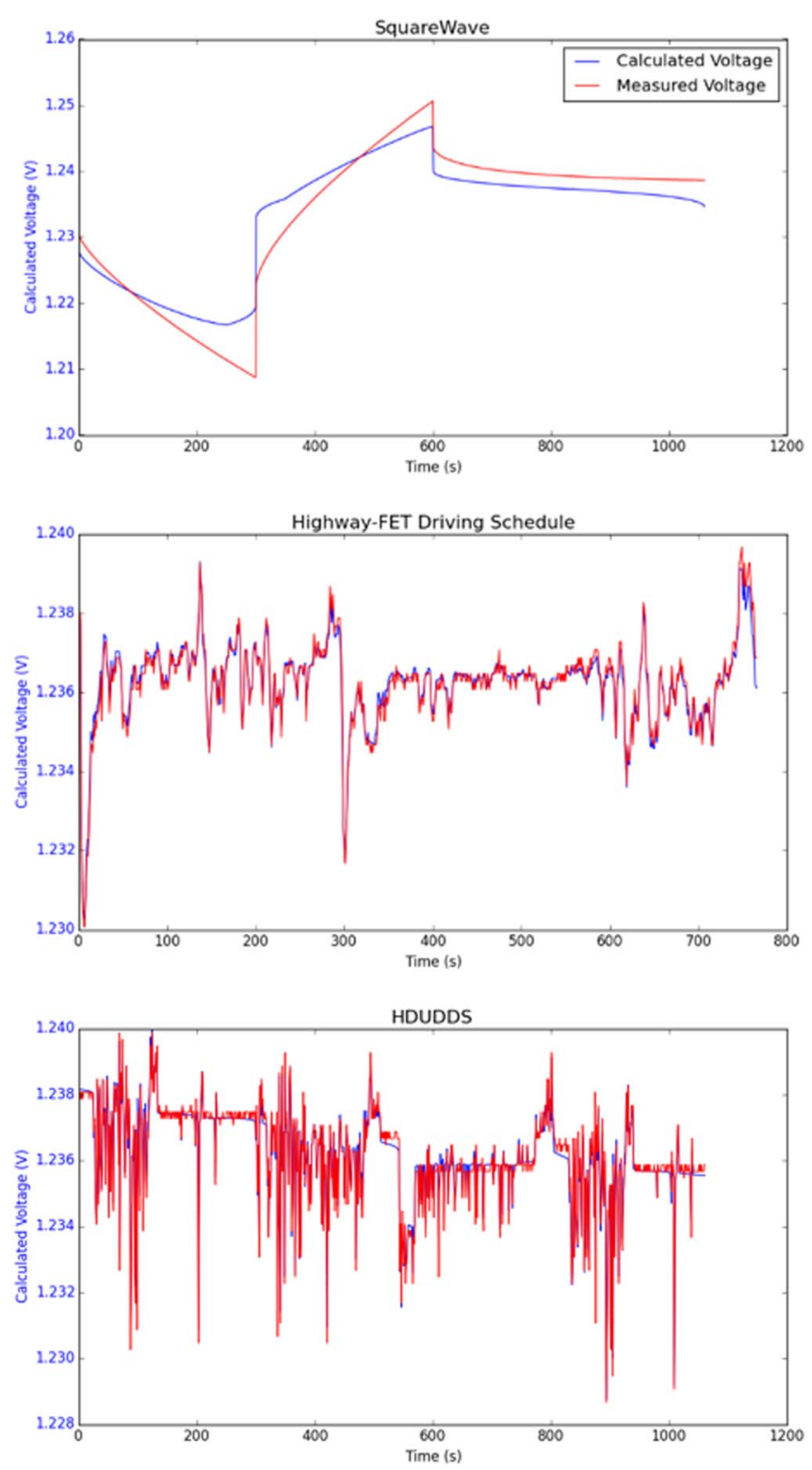

Figure 4. The response of NiMH (in red) and the predicted value (in blue) for three profiles at $120 \mathrm{~mA}$.

mechanisms, the approach described here is applicable to batteries of various chemistries as well as supercapacitors.

Discharge profile.-Three discharge profiles that are studied are chosen such that they have very fast moving parts in addition to relatively long and constant portions. Even though the approach works in a way that errors are less than $1 \%$ for all cases, as seen from the table, Squarewave is the one with the largest error. The main reason for this larger than others error is non-linearity of the fast moving part of the profile as explained in the next section.

Non-linearity.-One major assumption in the present report is that the system is linear. This assumption is warranted given the current algorithm relies heavily on the ability to measure a valid impedance spectrum of the system using the standard methods. Linearity of the system measured is an underlying assumption in doing any EIS measurement. ${ }^{20}$ The AC part of our approach cannot capture those nonlinearities that are not present in the EIS measurement, even though the DC part of the model does not have any linearity requirements.
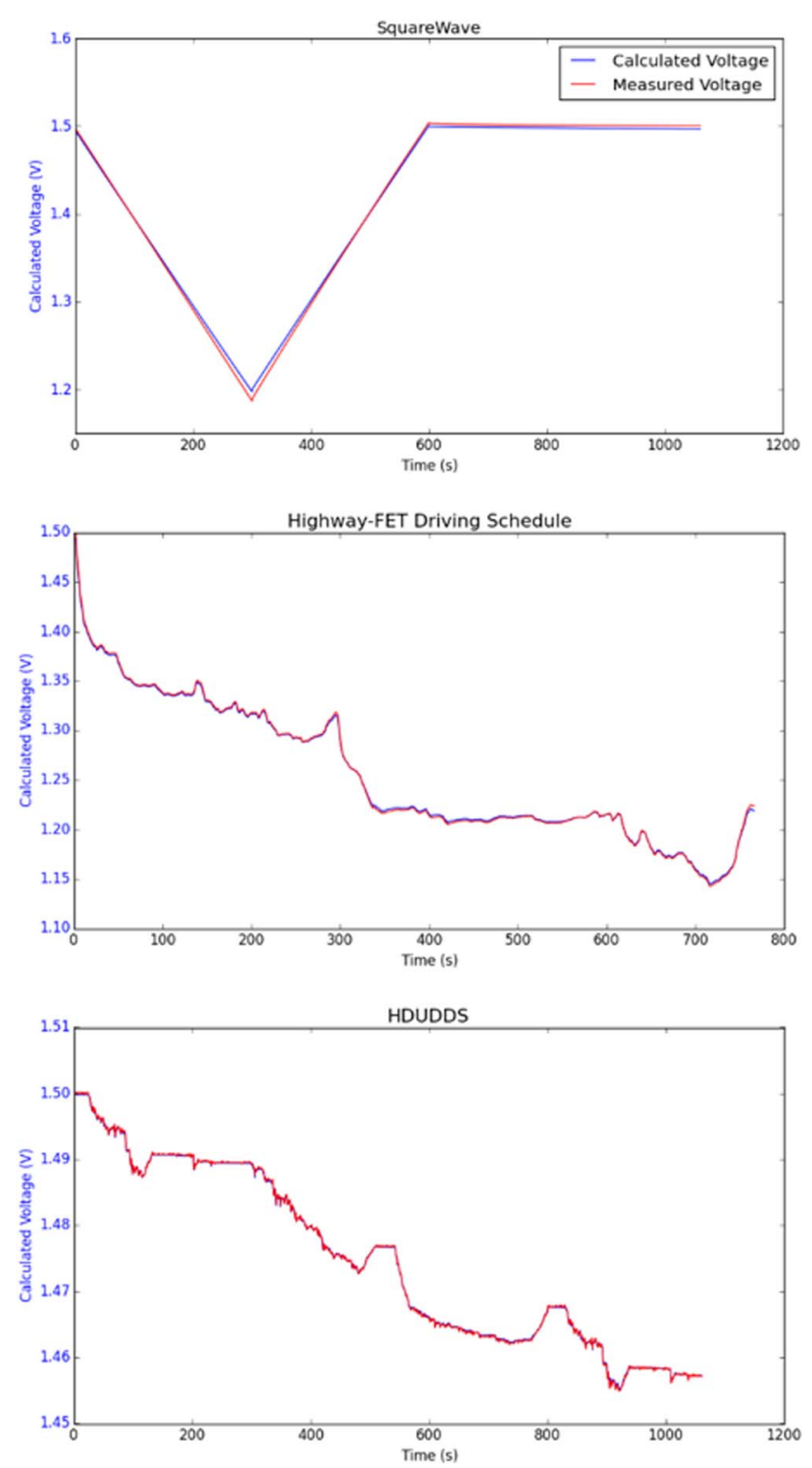

Figure 5. The response of $350 \mathrm{~F}$ (in red) and the predicted value (in blue) for three values at $350 \mathrm{~mA}$.

The frequency domain response of the profile that is used to test slow moving performance of the approach has a distinct peak in the frequency domain that dominates the response ${ }^{f}$. The amplitude at this frequency corresponds to a very large modulation that cannot be accurately explained with the linear EIS measurement that was done. This is the reason for the discrepancy that is higher than the other profiles which have a more uniform structure in the frequency domain. As a check for this phenomenon, Squarewave for LFP is shown in Figure 3. The error for this case is much smaller. For comparison, the Squarewave data for LFP at $220 \mathrm{~mA}$ is shown in supplementary information S3.

Variance of EIS characteristics with respect to SOC.-One major assumption in the modeling approach presented herein is that the impedance spectrum does not change appreciably through the period predicted. There are a number of reports in the literature that show that

${ }^{\mathrm{f}}$ At $1 / 600$ s frequency. This is simply because of the way the profile is constructed. Data not shown 

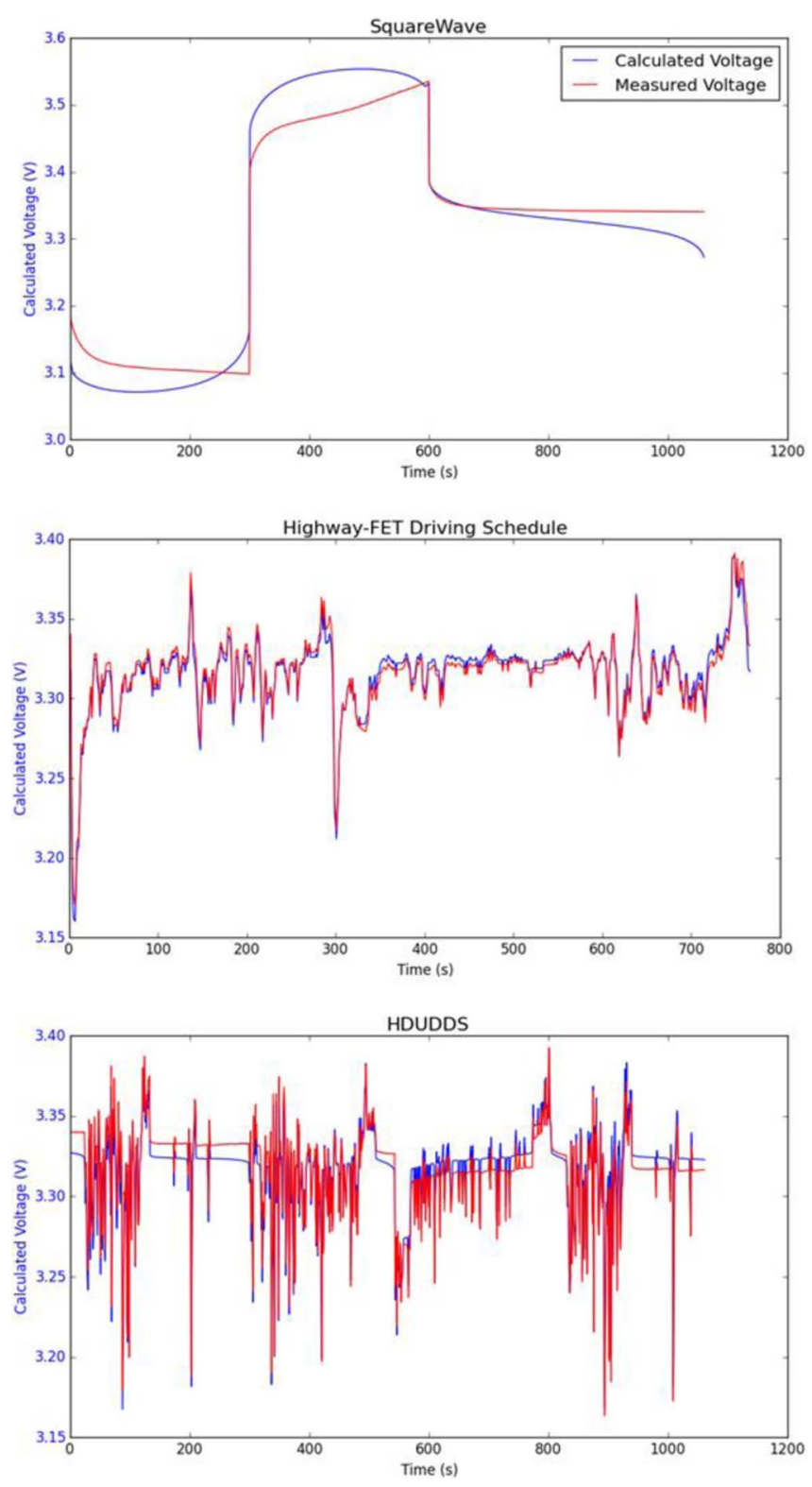

Figure 6. The response of LFP (in red) and the predicted value (in blue) for 2.2A discharge amplitude for all profiles used in this study. The maximum errors are less than $2.1 \%$ for Squarewave, $0.7 \%$ for Highway FET, $0.5 \%$ for HDUDDS.

the impedance spectrum varies measurably, albeit not greatly, with the SOC for certain chemistries. ${ }^{23,24}$ Though this variance was not significant in systems and the SOC tested within this work, it is conceivable that this is part of the reason for the observed error. In cases where this variance can potentially effect the results significantly, it can be taken into account using a SOC weighted average of the impedance spectra.

In order to confirm that this was not a problem in the present case, the tests were repeated at $3.12 \mathrm{~V}$ for the LFP cell. The data can be found in the supporting information figure S4. The errors are still all less than $0.1 \%$. This shows that in the present case, the above stated assumption is correct.

Performance of the approach at different amplitudes.-Tests were repeated at different amplitudes for three systems presented. Selected examples of those tests and the modeling performances are shown in the supporting information figure S3. None of the cases have more than $1 \%$ error. The data clearly show that, provided linear- ity is not compromised, the approach is valid across a wide range of amplitudes.

In order to test an edge-case scenario to find the limits of the modeling approach, the amplitude for the test was increased to 2.2A(i.e. 1C discharge rate) for the LFP sample. As shown in Figure 6, the predictions are still within $1 \%$ for Highway FET and HDUDDS, however, the error is $2.1 \%$ for the Squarewave signal. As mentioned above for NiMH cell, this is a problem with the nonlinearity of the response. As the signal in the frequency domain gets higher in amplitude, the contribution from the nonlinear parts of the response start becoming more significant. As mentioned above, this is particularly significant in the Squarewave case since the frequency domain counterpart of the current profile has a very large peak at the frequency $1 / 600 \mathrm{~s}$.

\section{Conclusions}

A new approach to modeling voltage behavior of batteries and supercapacitors is presented. This new approach is independent of battery chemistry or charge storage mechanism and relies on measurements of EIS and slow discharge. The approach is designed to be algebraically straightforward, with low computational complexity and with zero adjustable parameters. The described approach predicts the voltage response in all presented cases with less than $1 \%$ error in all and less than $0.3 \%$ in cases where linearity is maintained in the frequency domain.

\section{References}

1. S. F. Tie and C. W. Tan, A review of energy sources and energy management system in electric vehicles, Renew. Sustain. Energy Rev., 20, 82 (2013).

2. L. Zhang, B. Tiwana, Z. Qian, Z. Wang, L. Zhangt, R. P. Dickt, Z. Qiant, Z. M. Maot, R. P. Dick, Z. M. Mao, and L. Yang, Accurate online power estimation and automatic battery behavior based power model generation for smartphones, Proc. IEEE., 105 (2010).

3. A. Fotouhi, D. J. Auger, K. Propp, S. Longo, and M. Wild, A review on electric vehicle battery modelling: From Lithium-ion toward Lithium-Sulphur, Renew. Sustain. Energy Rev., 56, 1008 (2016).

4. S. Abada, G. Marlair, A. Lecocq, M. Petit, V. Sauvant-Moynot, and F. Huet, Safety focused modeling of lithium-ion batteries: A review, J. Power Sources., 306, 178 (2016).

5. Federal Aviation Administration, Lithium Batteries \& Lithium Battery Powered Devices, https://www.faa.gov/about/office_org/headquarters_offices/ash/ ash_programs/hazmat/aircarrier_info/media/battery_incident_chart.pdf/, 2017 (accessed 6.2.17)

6. National Transportation Safety Board, Interim Factual Report March 7, 2013. https://www.ntsb.gov/investigations/AccidentReports/Reports/DCA13IA037interim-factual-report.pdf/, 2017 (accessed 6.2.17).

7. D. Pavlov, Lead-acid Batteries, Science and Technology : a Handbook of Lead-acid Battery Technology and Its Influence on the Product. Elsevier Science \& Technology, 32 (2011).

8. P. Nelson, I. Bloom, K. Amine, and G. Henriksen, Design modeling of lithium-ion battery performance, 110, 437 (2002).

9. S. Nejad, D. T. Gladwin, and D. A. Stone, A systematic review of lumped-parameter equivalent circuit models for real-time estimation of lithium-ion battery states, $J$. Power Sources., 316, 183 (2016)

10. B. Šantić, A. Moguš-Milanković, and D. E. Day, The dc electrical conductivity of iron phosphate glasses, J. Non. Cryst. Solids., 296, 65 (2001).

11. J.Zhao, S. Aziz, and Y. Wang, Hierarchical functional layers on high-capacity lithiumexcess cathodes for superior lithium ion batteries, J. Power Sources., 247, 95 (2014).

12. S. Castano, L. Gauchia, E. Voncila, and J. Sanz, Dynamical modeling procedure of a Li-ion battery pack suitable for real-time applications, Energy Convers. Manag., 92, 396 (2015).

13. T. Osaka, T. Momma, D. Mukoyama, and H. Nara, Proposal of novel equivalent circuit for electrochemical impedance analysis of commercially available lithium ion battery, J. Power Sources., 205, 483 (2012).

14. E. Barsoukov, J. H. Kim, C. O. Yoon, and H. Lee, Universal battery parameterization to yield a non-linear equivalent circuit valid for battery simulation at arbitrary load, J. Power Sources., 83, 61 (1999).

15. G. Fan, K. Pan, M. Canova, J. Marcicki, and X. G. Yang, Modeling of Li-Ion Cells for Fast Simulation of High C-Rate and Low Temperature Operations, J. Electrochem. Soc., 163, A666 (2016).

16. M. Verbrugge, D. Baker, and X. Xiao, Formulation for the Treatment of Multiple Electrochemical Reactions and Associated Speciation for the Lithium-Silicon Electrode, J. Electrochem. Soc., 163, A262 (2016).

17. M. Thele, O. Bohlen, D. U. Sauer, and E. Karden, Development of a voltage-behavior model for NiMH batteries using an impedance-based modeling concept, J. Power Sources., 175, 635 (2008). 
18. M. Slocinski, Method for parameterization of impedance-based models with time domain data sets, Lect. Notes Impedance Spectrosc., 4, 3 (2013)

19. United States Environmental Protection Agency, Dynamometer Drive Schedules. https://www.epa.gov/vehicle-and-fuel-emissions-testing/dynamometer-driveschedules/, 2017 (accessed 5.10.15).

20. United States Environmental Protection Agency, Highway Fuel Economy Test Drive Schedule. https://www.epa.gov/sites/production/files/2015-10/hwycol.txt/, 2017 (accessed 5.10.15)
21. United States Environmental Protection Agency, Heavy Duty Urban Dynamometer Drive Schedule. https://www.epa.gov/sites/production/files/2015-10/huddscol.txt/, 2017 (accessed 5.10.15)

22. M. Orazem and B. Tribollet, Electrochemical Impedance Spectroscopy, Wiley; 2008

23. S. Rodrigues, N. Munichandraiah, and A. K. Shukla, Review of state-of-charge indication of batteries by means of a.c. impedance measurements, J. Power Sources., 87, 12 (2000).

24. S. Piller, M. Perrin, and A. Jossen, Methods for state-of-charge determination and their applications, J. Power Sources., 96, 113 (2001). 